Opinion

\section{Biological membranes: The laboratory of fundamental physics}

\author{
Samo Kralj1* and Mitja Kralj² \\ 'Faculty of Natural Sciences and Mathematics, University of Maribor, Koroska 160, 2000 Maribor, \\ Slovenia \\ ${ }^{2}$ SKUC, Stari trg 21, Ljubljana, Slovenia
}

Biological membranes present an essential constituent of living cells. Their main role is to separate the interior of a cell from its surrounding, however allowing the selective transfer of specific material through it. Configuration changes of membranes are often correlated with important biological processes [1-7]. For example, they might trigger divisions of cells [4], adaptation of red blood cells [1] to temporal conditions during their transport to different parts of biological tissues, they might be involved in cancerous [5] and cell death [6] processes... Membrane structures are in general extremely complex, however, their key properties are often dominated by geometry. This was first illustrated by Helfrich [8] who constructed a minimal model of membranes introducing curvature fields. Locally, these are represented by principal curvatures $C_{1}=1 / R_{1}$ and $C_{2}=1 / R_{2}$, where $R_{1}$ and $R_{2}$ are the corresponding curvature radii. The key quantities dominating energetics of membranes are the mean curvature $H=\left(C_{1}+C_{2}\right) / 2$ and the Gaussian curvature $K=C_{1} C_{2}$. In general, membranes tend to minimize $H^{2}$ for given boundary conditions and $K$ plays an important role if a membrane undergoes a topological change (i.e. in membrane fission or fusion processes). Furthermore, membranes in general often exhibit some kind of in-plane order, which enormously increases the complexity of potential membrane responses to various stimuli. This ordering could be due to anisotropic membrane constituents [9], flexible hydrocarbon chains of lipids [10] or due to anisotropic proteins embedded within membranes [11]. If in-plane order exists topological defects (TDs) [12] are inevitably formed in membranes if they do not exhibit toroidal symmetry [13]. TDs in membranes correspond to points or lines where the in-plane field is (mathematically) not uniquely defined as illustrated in figure 1. Consequently, such regions are in general energetically costly. In practice, membranes avoid such singularities by locally "melting" [4] of in-plane order or by a local phase separation [9]. The former case corresponds to relatively strong local fluctuations, via which the in-plane ordering is averaged out. In the latter case a membrane ingredient responsible for anisotropic ordering moves to "nonsingular" membrane parts.

\section{More Information}

*Address for Correspondence: Samo Kralj, FNM, University of Maribor, Koroska 160, 2000 Maribor, Slovenia, Tel:+38631389278; Email: samo.kralj@um.si

Submitted: 19 October 2019

Approved: 31 October 2019

Published: 01 November 2019

How to cite this article: Kralj S, Kralj M. Biological membranes: The laboratory of fundamental physics. Int J Phys Res Appl. 2019; 2: 038-040. DOI: dx.doi.org/10.29328/journal.ijpra.1001013

Copyright: @ 2019 Kralj S, et al. This is an open access article distributed under the Creative Commons Attribution License, which permits unrestricted use, distribution, and reproduction in any medium, provided the original work is properly cited.

\section{Check for updates}

One can assign to TDs a topological charge, which is a conserved quantity. If membranes are treated as effectively two-dimensional objects, the topological charge equals [12] the winding number $m$. The later determines the total reorientation of the in-plane field divided by $2 \pi$ on encircling the defect center counterclockwise. Examples if figure 1 represent TDs bearing charges $m=1$ (Figure 1a) and $m=-1$ (Figure 1b). In general, TDs behave like localized electric charges, where $m$ plays the role of an electrical charge. Furthermore, TDs are energetically costly and flat parts of the membrane tend to expel them. However, in closed membranes, their total winding number $m_{\text {tot }}$ is determined topologically. Namely, it holds [14].

$$
\mathrm{m}_{\text {tot }}=\frac{1}{2 \pi} \oint K \mathrm{da}
$$

where the integral is carried over the closed membrane and $d a$ stands for an infinitesimally small surface area. For example, for the spherical (toroidal) topology it holds $m_{\text {tot }}$ $2\left(m_{\text {tot }}=0\right)$. Furthermore, in "normal" (relatively weak curvatures) conditions "elementary" TDs tend to be formed.

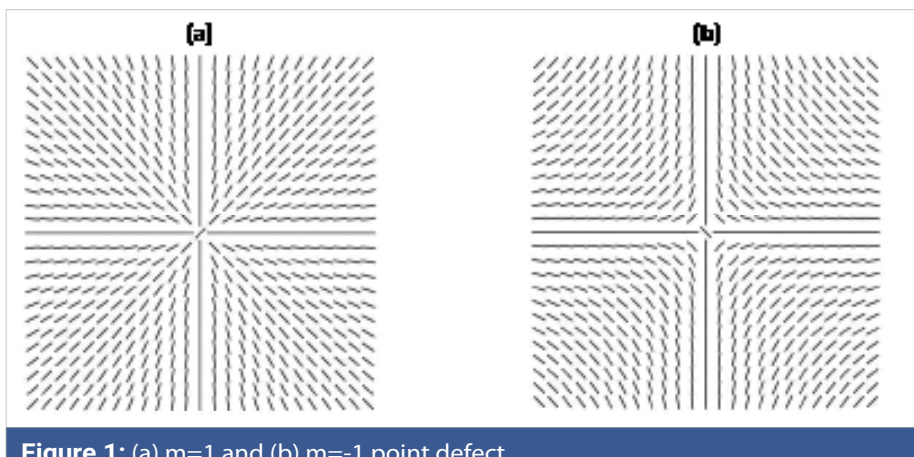


In case of i) vector, ii) rod-like, or iii) hexatic order these TDs carry winding numbers i) $m_{0}= \pm 1$, ii) $m_{0}= \pm 1 / 2$, and iii) $m_{0}= \pm 1 / 6$. Therefore, a membrane of spherical topology exhibits at least i) two, ii) four, and iii) twelve TDs. One could intuitively understand this tendency by inspecting figure 2 . Let us assume that an in-plane ordering exists, which tends to be locally parallel. In the case of toroidal topology, the field could stream along the parallels (lines parallel to the equatorial line). Such topology does not impose frustration on orientational order and does not require any TDs figure 2a. However, for a spherical topology TDs are unavoidable because the geometry enforces frustration to the orientational field. In figure $2 \mathrm{~b}$ one sees that two $m=1$ defects are formed at the poles of the structure. Eq.(1) also suggests that positive (negative) Gaussian curvature attracts TDs bearing positive (negative) values of $m[13,15]$, (i.e. $d m_{\text {tot }}=K d a / 2 \pi$ ). This is efficiently realized in regions with $K \neq 0$ where a difference between the principal curvatures is relatively small. However, if this is not the case, the difference imposes a kind of local field, referred to as the deviatoric field [16]. Consequently, TDs tend to be expelled from such regions because their spatially non-uniform structure is incompatible with the field imposed uniform ordering. Note that TDs introduce local inhomogeneities in membranes, which could serve as nucleating regions for various biological (e.g., membrane fission or budding) processes $[3,4]$. To conclude, membranes in general host of TDs and their positions and also the number is strongly affected by curvature fields. Furthermore, TDs in membranes could play an important role in their functionality.

Related problems are also of strong interest for other branches of condensed matter physics, and even for cosmology and particle physics. Namely, TDs are an unavoidable consequence of symmetry-breaking phase transitions [12], which are ubiquitous in nature.

Furthermore, their main properties are determined topologically, which are independent of systems' microscopic details, endowing them with several universal features. For example, in general, the impact of curvature on TDs in twodimensional systems is relatively weakly understood in condensed matter systems. Namely, most theoretical studies [a]

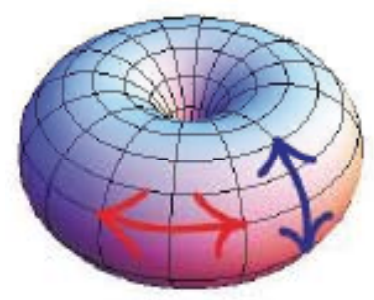

b)

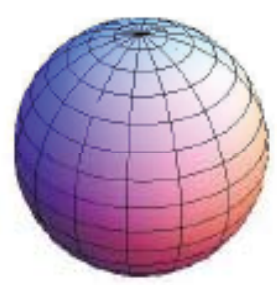

Figure 2: (a) Toroidal topology. The red double arrow indicates a parallel, and blue colored arrow a meridian. (b) Spherical topology. If an ordering field is aligned either along meridians or parallels it exhibits point defects at the poles.
$[14,15]$ use covariant derivatives in expressing the elastic penalty of distorted ordering fields. Such approaches by default discard the so-called extrinsic curvature contributions [14] and take into account only intrinsic contributions. However, there are no reasonable justifications $[17,18]$ for this omission. Simple analysis [17] even suggests that the extrinsic and intrinsic contributions should be comparable and in several geometries, they enforce contradicting behaviour $[17,18]$. Therefore, by omitting extrinsic contributions several extrinsic curvature-driven phenomena could not be observed. Note that the extrinsic curvature is in biological systems commonly referred to as the deviatoric curvature and its impact on various membrane properties has been relatively well explored [9]. Therefore, other branches of physics could transfer some of this knowledge to their realm. Furthermore, the extrinsic curvature exhibits the impact of the higher dimensional space in which the lower-dimensional system of interest is embedded (i.e., effectively two-dimensional curved membranes are embedded in three dimensions). Therefore, extrinsic curvaturedriven phenomena could reveal the impacts of potentially existing higher dimensions, which is of interest in cosmology. Furthermore, the first theory of coarsening dynamics (the so-called Kibble-Zurek mechanism [19]) of TDs following a sudden phase transition was developed in cosmology to study the coarsening of TDs in the Higgs field of the early universe [20]. Therefore, some of this knowledge might be transferred also to membranes. Note that interactions between curvature and TDs might even resolve the origin of mysterious dark energy. Namely, the mainstream description of nature is based on the assumption that the universe is essentially flat. However, recent numerical studies [21] reveal that the current universe could exhibit finite curvature. By taking into account the impact of curvature one could reproduce effects, which are now attributed to the mysterious dark energy. Moreover, if relevant fields represent basic entities of nature [22], then TDs [23] might represent fundamental particles in cosmology's Standard Model terminology...

\section{References}

1. Deuticke B. Transformation and restoration of biconcave shape of human erythrocytes induced by amphiphilic agents and changes of ionic environment. Biochim. Biophys. Acta. 1968; 163: 494-500. PubMed: https://www.ncbi.nlm.nih.gov/pubmed/4387277

2. Iglič A, Babnik B, Gimsa $U$, Kralj-Iglič V. On the role of membrane anisotropy in the beading transition of undulated tubular membrane structures. J Phys A Math Gen. 2006; 38: 8527.

3. Hurley JH, Boura E, Carlson LA, Różycki B. Membrane Budding. Cell. 2010; 143: 875-887.

PubMed: https://www.ncbi.nlm.nih.gov/pubmed/21145455

4. Jesenek D, Perutková S, Kralj-Iglič V, Kralj S, Iglič A. Exocytotic fusion pore stability and topological defects in the membrane with orientational degree of ordering. Cell Calcium. 2012; 52: 277-282.

PubMed: https://www.ncbi.nlm.nih.gov/pubmed/22541648

5. Lee J, Abdeen AA, Wycislo KL, Fan TM, Kilian KA. Interfacial geometry dictates cancer cell tumorigenicity. Nat Mater. 2016; 15: 856-862.

PubMed: https://www.ncbi.nlm.nih.gov/pubmed/27043781 
6. Saw TB, Doostmohammadi A, Nier V, Kocgozlu L, Thampi S, et al Topological defects in epithelia govern cell death and extrusion. Nature. 2017; 544: 212-216.

PubMed: https://www.ncbi.nlm.nih.gov/pubmed/28406198

7. Kumar G, Ramakrishnan N2, Sain A. Tubulation pattern of membrane vesicles coated with biofilaments. Phys Rev E. 2019; 99: 022414.

PubMed: https://www.ncbi.nlm.nih.gov/pubmed/30934309

8. Helfrich W. Elastic properties of lipid bilayers: theory and possible experiments. Zeits Naturforschung. 1973; 28: 693-703.

PubMed: https://www.ncbi.nlm.nih.gov/pubmed/4273690

9. Kralj-Iglič V, Heinrich V, Svetina S, Žekš B. Free energy of closed membrane with anisotropic inclusions. Eur Phys J B. 1999; 10: 5-8.

10. Perutková Š, Daniel M, Rappolt M, Pabst G, Dolinar G, et al., Elastic deformations in hexagonal phases studied by small-angle $\mathrm{X}$-ray diffraction and simulations. Phys Chem Chem Phys. 2011; 13: 3100-3107. PubMed: https://www.ncbi.nlm.nih.gov/pubmed/21063616

11. Fournier JB. Nontopological saddle-splay and curvature instabilities from anisotropic membrane inclusions. Phys Rev Lett. 1996; 76: 4436-4439. PubMed: https://www.ncbi.nlm.nih.gov/pubmed/10061289

12. Mermin ND. The topological theory of defects in ordered media. Rev Mod Phys. 1979; 51: 591.

13. Mesarec L, Góźdź W, Iglič A, Kralj S. Effective topological charge cancelation mechanism. Sci Rep. 2016; 6: 27117.

14. Kamien RD. The topological theory of defects in ordered media. Rev Mod Phys. 2002; 74: 953.
15. Bowick M, Nelson DR, Travesset A. Curvature-induced defect unbinding in toroidal geometries. Phys Rev E. 2004; 69: 041102.

16. Kralj-Iglic V, Iglic A, Hägerstrand $H$, Peterlin P. Stable tubular microexovesicles of the erythrocyte membrane induced by dimeric amphiphiles. Phys Rev E. 2000; 61: 4230.

PubMed: https://www.ncbi.nlm.nih.gov/pubmed/11088219

17. Selinger RL, Konya A, Travesset A, Selinger JV. Monte Carlo studies of the $X Y$ model on two-dimensional curved surfaces. J Phys Chem B. $2011 ; 115: 13989-13993$.

PubMed: https://www.ncbi.nlm.nih.gov/pubmed/21970652

18. Napoli G, Vergori L. Extrinsic curvature effects on nematic shells. Phys Rev Lett. 2012; 108: 207803.

PubMed: https://www.ncbi.nlm.nih.gov/pubmed/23003189

19. Kibble TWB. Topology of cosmic domains and strings. J Phys A Math Gen. 1976; 9: 1387.

20. Zurek WH. Cosmological experiments in superfluid helium? Nature. 1985; 317: 505.

21. Giblin JT, Mertens JB, Starkman GD. Departures from the FriedmannLemaitre-Robertson-Walker Cosmological Model in an Inhomogeneous Universe: A numerical Examination. Phys Rev Lett. 2016; 116: 251301. PubMed: https://www.ncbi.nlm.nih.gov/pubmed/27391710

22. Hobson A. There are no particles, there are only fields. Am J Phys. 2013; 81: 211-223.

23. Skyrme T. A unified field theory of mesons and baryons. Nucl Phys. 1962; 31: 556-559. 